\title{
Réflexions sur les Facteurs Déterminants de l'Évolution du Financement de l'Enseignement Supérieur au Canada dans les Années 1970 et 1980.
}

\author{
Pierre-Paul Proulx*
}

\section{INTRODUCTION}

Nous sommes tous conscients que l'enseignement supérieur et la recherche universitaire, au Canada, traversent une période critique marquée particulièrement par l'arrivée sur le marché du travail d'un surplus de diplômés, partout sauf au niveau des deuxième et troisième cycles du secteur francophone du Québec. Ce taux de croissance élevé du nombre de diplômés a provoqué l'apparition de restrictions financières qui ralentiront le rythme de changement dans le système d'enseignement.

Ce texte aborde un ensemble de facteurs qui affectent actuellement le financement de l'enseignement supérieur et dont les effets pourraient se poursuivre bien au-delà de 1980. Il est vrai qu'on pourrait assister, d'ici 1980, à des modifications relativement importantes du système d'enseignement, mais il me semble que beaucoup de changements seront surtout de nature qualitative, donc moins apparents que ceux observés ces dernières années. D'autre part je crois qu'il s'effectuera moins de transformations d'ici 1980 et qu'il faudra attendre au-delà de cette date pour constater la réalisation des mutation discutées maintenant.

D'ailleurs, la reconduction pour trois années de la formule actuelle de financement de l'enseignement post-secondaire ne favorise pas certains changements importants. Nous en sommes, en fait, à discuter des modalités d'application des propos élaborés longuement dans le Rapport Faure, dans le rapport ontarien «The Learning Society 》, dans le rapport du Conseil des Universités sur «Les objectifs généraux de l'enseignement supérieur », dans « A Choice of Future », dans le Rapport Hall-Dennis, etc...

* Pierre-Paul Proulx, Directeur, Centre de Recherches en Développement Économique, Université de Montréal. 
Nous présenterons d'abord quelques réflexions générales sur le sujet pour ensuite examiner les facteurs d'offre et de demande qui affecteront le financement de l'enseignement supérieur, et nous conclurons par une brève discussion des changements majeurs en perspective.

\section{REFLEXIONS GENERALES}

Puisque le système d'enseignement post-secondaire évolue plus ou moins au même rythme que la société, c'est par une discussion des facteurs qui affectent cette évolution que nous allons aborder notre sujet, pour ensuite revenir aux modifications du contenu, des méthodes et de diverses autres modalités du système d'enseignement post-secondaire.

Il semble acquis que des facteurs de nature centripète et centrifuge, entendu ici dans le sens de facteurs respectivement orientés vers l'Homme en tant qu'individu et vers les problèmes à portée supranationale, ne cessent d'affecter l'évolution socio-culturelle de la société, donc l'évolution de la nature et du contenu du système d'enseignement supérieur et, par ricochet, celle de son financement.

Les facteurs d'évolution de nature centripète proviennent, croyons-nous, de ce que les activités économiques se concentrent de plus en plus dans le secteur tertiaire, entraînant ainsi des contacts plus nombreux et plus soutenus entre les individus. Nous irions donc vers une ère où ces individus chercheraient à vivre au sein de groupes ayant des objectifs communautaires, à participer aux décisions qui les affectent et à associer éducation et travail, enseignement et vie. Ainsi, le contenu de la formation et les méthodes de l'acquérir porteraient davantage sur les aspects esthétiques, physiques, intellectuels, professionnels et culturels de leur existence, ce qui implique une décentralisation beaucoup plus poussée des modes de planification, de gestion et de financement de la formation ainsi qu'une plus grande part de formation autodidacte et de formation par la recherche. Autrement dit, c'est parler de ce qu'on nomme souvent l'éducation permanente.

Par ailleurs, ce désir d'associer enseignement et vie joint au taux élevé de natalité de l'après-guerre dont les effets se répercutent aujourd'hui sur le marché du travail, me porte à croire que la formation sera de plus en plus dispensée hors du système formel d'enseignement, comme c'est le cas actuellement pour la formation dispensée par les entreprises. En conséquence, on doit cesser d'envisager la formation post-secondaire dans le cadre strict du système actuel d'enseignement et se pencher, de façon plus réaliste, sur le financement de la formation offerte dans le cadre des politiques de main-d'œuvre, comme les programmes d'Initiatives Locales, de Perspectives Jeunesse, etc... Illich et McLuhan avaient pressenti ce mouvement vers une déformalisation et une désinstitutionalisation de l'apprentissage, mouvement où la pédagogie d'enseignement utilise plus largement la télévision communautaire, les films, les centres de documentation et d'auto-enseignement à l'aide de calculatrices, etc... 
Aux facteurs centripètes s'ajoutent des facteurs centrifuges illustrés, en particulier, par des problèmes comme:

a) l'océan et ses ressources ;

b) l'énergie et sa disponibilité ;

c) les ressources naturelles rares et leur gestion ;

d) la climatologie, les communications internationales et les divers aspects de la pollution et leur contrôle ; etc...

Ce sont là des questions qui ne peuvent être traitées par des gouvernements isolés puisqu'elles impliquent, de plus en plus, des travaux et des échanges internationaux et, partant, des molifications de structure, des communications accrues et un contenu très différent de connaissances à découvrir et à assimiler.

Enfin, le caractère de plus en plus public du financement de l'enseignement supérieur constitue une contrainte pour des gouvernements dont les priorités s'orientent vers les disparités ethniques et régionales, vers le bien-être, vers la création d'emplois, provoquant ainsi des effets négatifs sur les sommes disponibles pour le financement de l'enseignement post-secondaire. Effectivement ces changements de priorités, d'une part, et l'état actuellement peu avancé de la planification de l'enseignement supérieur d'autre part, auront peut-être pour effet d'engendrer des pénuries de main-d'œuvre hautement qualifiée vers 1980. Cependant, il importe d'analyser, en détail, les multiples facteurs susceptibles d'affecter cette siuation d'offre et de demande de main-d'œuvre hautement qualifiée. Nous ne pourrons y arriver dans le cadre de cette étude, mais passons quand même en revue certains facteurs qui nous semblent pertinents.

\section{FACTEURS D'OFFRE ET LEURS EFFETS SUR LE FINANCEMENT DE L'ENSEIGNEMENT SUPERIEUR}

Certains facteurs déterminants du nombre d'étudiants qui recevront un enseignement post-secondaire, ont des effets contraires. Ainsi, l'objectif d'accessibilité de plus en plus concrétisé par diverses décisions gouvernementales tend à faire croître l'offre d'étudiants, tandis que l'explosion démographique d'après-guerre, dont la vague principale est sortie du système actuel d'enseignement, le phénomène des drop-outs, etc., peuvent entraîner une diminution de l'offre d'étudiants dans le système formel d'enseignement. ce qui concerne :

L'objectif d'accessibilité se manifeste surtout par une préoccupation croissante en

a) le rôle et la participation des femmes sur le marché du travail ;

b) la redistribution des revenus;

c) la pauvreté ;

d) les amérindiens; 
e) les francophones;

f) l'accès spatial plus équilibré aux système d'enseignement d'où des effets positifs sur le financement de l'enseignement post-secondaire.

Enfin, l'importance croissante du phénomène des drop-outs qui reflète, semble-t-il, un rejet de la formule de la formation séquentielle, entraînera, par la suite, l'apparition d'un phénomène de drop-ins. Ce dernier phénomène, ajouté à l'implantation graduelle des horaires de travail flexibles, accentuera la tendance actuelle vers une formation en plusieurs phases, modifiant ainsi la structure d'âge des étudiants et nécessitant une plus grande souplesse dans les politiques d'administration et dans la programmation des enseignements.

Certains de ces changements peuvent stimuler l'intérêt pour la formation dans le cadre des entreprises de sorte que le développement des politiques de main-d'œuvre se fera aux dépens de l'enseignement supérieur formel.

\section{FACTEURS DE DEMANDE ET LEURS EFFETS SUR LE FINANCEMENT DE L'ENSEIGNEMENT SUPERIEUR}

De nombreux facteurs peuvent affecter la demande de diplômés. Entre autres, le rôle des corporations professionnelles dans la création de postes pour des techniciens dans diverses professions peut faire naître de nouveaux programmes d'enseignement dans ce domaine. De plus, une diminution de l'importance relative accordée aux études de deuxième et troisième cycles, sauf au Québec, est de nature à réduire l'augmentation des coûts de l'enseignement. Enfin, l'engouement pour l'innovation peut susciter des fonds pour des projets de nature expérimentale.

On a longtemps exagéré, à mon avis, la contribution de l'éducation comme facteur de croissance économique, d'autant plus que cette contribution est lente à se manifester. A l'avenir, semble-t-il, c'est l'évolution du Produit National Brut qui constituera le facteur déterminant du financement de l'enseignement. C'est pourquoi, sans vouloir jouer au prophète, on peut prévoir que la faiblesse du taux de croissance du Produit National Brut aux cours des dernières années, malgré une reprise récente, et la faiblesse du taux de rendement de la formation ainsi que le surplus de main-d'œuvre, donneront lieu à des restrictions financières considérables. En contrepartie, il ne serait pas surprenant de voir augmenter le niveau des ressources financières disponibles pour les programmes de formation dispensée en dehors des cadres traditionnels.

D'autre part, on pourrait également prévoir que les politiques de développement industriel et les politiques scientifiques actuelles au Canada auraient pour effet d'augmenter la demande de diplômés. Autrement dit, l'intérêt porté aux problèmes d'envergure internationale ne manquerait pas d'influencer la politique scientifique canadienne et de libérer des fonds pour la recherche universitaire. Par contre, étant donné que les ententes sur le financement de l'enseignement post-secondaire ne favorisent pas la recherche 
dans les universités et que ces ententes ont été reconduites pour trois ans et, étant donné le fait que les nouvelles politiques scientifiques semblent privilégier la recherche industrielle, on risque de voir augmenter les forids disponibles pour ce secteur. Il est vrai, cependant, qu'une bonne partie de ce fonds est acheminée vers les universités sous forme de sous-contrats.

\section{CONCLUSIONS}

Je partage entièrement la vision qui se dégage du Rapport Faure, bâti sur un ensemble de réflexions, de travaux et de rapports, et qui conclut que nous allons vers un système d'enseignement global et ouvert, utilisant une multitude de moyens, le tout axé principalement sur l'individu. En conséquence, nous nous orientons, semble-t-il, vers des systèmes décentralisés de gestion et de décision où la formation sera, de plus en plus, dispensée hors du système formel d'enseignement.

De plus, ces mutations entraîneront une variété considérable de points d'entrée et de re-entrée dans les programmes de formation, forçańt ainsi la recherche de formules de financement flexibles et adaptés à la complexité de ce que seront les prévisions de ces points d'entrée et de re-entrée. Dans un tel cas, il sera préférable d'adopter une formule de financement donnant plus d'emphase au financement via les individus au moyen de prêts et de bourses qu'aux institutions, d'autant plus que la première est plus facile à administrer dans le cadre d'une politique d'accessibilité.

On doit également tenir compte de la possibilité de poursuite simultanée de travail et d'éducation qui, si elle se manifeste de façon plus concrète, fera en sorte d'augmenter la capacité de payer de ceux qui seront aux études, entraînant ainsi une baisse relative de la part publique dans le financement de l'enseignement supérieur.

Enfin, toutes ces réflexions sur les facteurs déterminants de l'évolution du financement de l'enseignement post-secondaire ne permettent pas de tirer une conclusion nette et claire sur le sujet. Je reste, cependant, frappé par certains mouvements de fond, repris de façon très générale dans de multiples rapports dont j’ai pu prendre connaissance récemment et qui rejoignent certaines tendances que $j$ 'ai tenté de faire ressortir dans ce texte.

\section{CITATIONS}

«Une révolution s'annonce. Elle ne sera pas comme les révolutions du passé. Elle tire ses origines dans l'individu et sa culture, et la structure politique ne sera changée qu'en dernier lieu. Elle ne nécessite pas de violence pour réussir, et elle ne peut être arrêtée par la violence. Cette révolution se répand avec une rapidité effarante et, déjà, nos lois et institutions et le contexte social s'en voient modifiés. Elle apporte une communauté plus humaine et un individu renouvelé et plus libre. Le résultat ultime sera l'apparition d'une nouvelle relation entre homme et hommes, homme et nature, et homme et société. 》 
C.A. Reich, The Greening of America; Penguin Books, 1970, p. 11 (traduction de l'auteur).

«Le paradoxe, c'est que dans une société vouée aux changements rapides, l'école doit préparer à un monde difficilement prévisible ; l'accent doit donc être mis, non sur la transmission des connaissances, mais sur la capacité d'adaptation et sur l'imagination. »

François Cloutier au Symposium sur le Rapport Faure organisé par la Commission Canadienne pour l'UNESCO, le 12 avril 1973.

«Un système d'enseignement post-secondaire visant à entrevoir l'accessibilité plus complète doit rechercher comme objectif la diversité. Ceci implique plus de choix dans la nature de la formation disponible. On peut donc choisir entre divers contenus: sciences humaines, formation professionnelle, humanité, arts. Il faut aussi choisir entre diverses méthodes pédagogiques: cours magistraux, séminaires, rencontres individuelles, correspondance, calculatrice, audio-visuel. De plus, il y a le lieu à choisir, car l'apprentissage peut se faire à domicile, sur le temps du travail ou dans une institution. D'autre part, il y a des objectifs à préciser, à savoir, ce peut être la formation personnelle, la formation en vue d'une carrière, la connaissance superficielle dans un domaine du savoir, ou au contraire une connaissance approfondie d'un secteur. Il y a aussi à choisir entre divers modes d'apprentissage: à temps partiel, à temps plein, de façon séquentielle ou interrompue. De plus, il y a nécessité de choisir le temps: le jour, la nuit, l'hiver, l'été, à l'année ou en tout temps pour l'autodidacte. »

The Learning Society, Report of the Commission on Post-Secondary Education in Ontario, Ministry of Government Services, Toronto, 1972, p. 41.

« Principe organisateur de toute éducation, elle (l'éducation permanente) implique un système complet, cohérent et intégré, offrant les moyens propres à répondre aux aspirations d'ordre éducatif et culturel de chaque individu et conformes à ses facultés. Elle est destinée à permettre à chacun de développer sa personnalité toute sa vie durant, par son travail ou par ses activités de loisir. Elle est également déterminée par les responsabilités que chaque individu a envers la société. »

Conseil de l'Europe, cité par le Conseil des Universités, « Objectifs généraux de l'enseignement supérieur et orientation des établissements 》; Cahier 2, Québec, le 22 février 1973, p. 4. 